\title{
The Impact of User Heterogeneity on Knowledge Collaboration Performance
}

\author{
Yingying ZHOU, Jianbin $\mathrm{CHEN}^{*}$, Shuli GAO, Mo CHEN
}

\begin{abstract}
Existing research showed that the social $Q \& A$ community is centred on user participation and can improve knowledge collaboration performance by means of heterogeneous knowledge, but it did not reveal the process and mechanism. This paper takes the user behaviours of the Zhihu which is the largest social $Q$ \& A community in China as the research object. From an interactive perspective, it builds a research model of the relationship between user heterogeneity and knowledge collaboration performance by establishing measurement indicators for relationship analysis according to the user behaviours data of Zhihu. The results show that there is an inverted $U$ shaped relationship between user heterogeneity and knowledge collaboration performance, and the interpersonal interaction of users plays a mediating role in the relationship between user heterogeneity and knowledge collaboration performance, while the mediating effect of machine interaction is not significant. This paper, based on this, puts forward some suggestions for better development of social $Q$ \& A community.
\end{abstract}

Keywords: interactivity; knowledge collaboration performance; social Q \& A community; user heterogeneity

\section{INTRODUCTION}

In the context of social media, the rapid iteration of internet technology and the development of the fragmented knowledge economy have promoted the diversification of knowledge sharing methods. The traditional question-andanswer community (Q \& A community) in the Web 1.0 era only focused on the value-added knowledge capital, while little attention was paid to the social capital, i.e., interaction between users, resulting in loose community relations and a high rate of user churn. The social Q \& A community in the Web 2.0 era represented by the Zhihu effectively made up for this defect, emphasizing the interaction between users, forming a close social network between users and users, users and problems, and users and topics. It has made the sharing and discussion of knowledge and information more in-depth, and the relationship between community users has been strengthened, and sustainable development community ecology has been constructed, in which knowledge capital and social capital have grown simultaneously.

This relies on the inherent network externalities of the virtual community, allowing users to gather quickly based on a certain topic or knowledge field, and user heterogeneity continues to expand [1]. Although there is a certain degree of heterogeneity among members of the socialized Q \& A community at this stage, there are still some issues that need to be clarified, such as the mechanism by which user heterogeneity is converted into knowledge collaboration performance. And what are the reasons for high user silence and low knowledge interaction performance?

Innovation comes from the recombination of different knowledge, information, and experience. Heterogeneous information and knowledge form the basis of innovation [2]. Knowledge collaboration is the process of creating new knowledge through different combinations of knowledge resources. At the enterprise level, its innovation performance depends not only on the degree of heterogeneity of knowledge resources, but also is directly affected by the enterprise's learning methods and innovation strategies [3]; at the level of Q \& A community, its knowledge interaction performance depends not only on the degree of heterogeneity of user knowledge, but also on the user's activity in the community, namely, interactivity [4]. The basic function of the social Q \& A community is to gather heterogeneous knowledge subjects, while the essence is user interaction, not a simple knowledge collection, which is recognized by the interactive team of Cooke et al. [5] (Interactive Team Cognition, referred to as ITC) that theories are similar in view, which emphasize that the cooperative activities of heterogeneous members in coordination, communication and decision-making are fundamental to promote the evolution of the team's cognitive system.

At present, many scholars have analysed social Q \& A communities from the aspects of community management [6], user participation motivation [7], and community topics [8], while few explore the relationship between user heterogeneity and knowledge collaboration performance from an interactive perspective. In addition, the research on user heterogeneity and knowledge collaboration performance is mainly based on static and questionnaire data, ignoring the dynamic changes of users, and cannot avoid the high error between the questionnaire's subjective expression and actual behaviours. In view of this, based on the objective user behaviours data of Zhihu, which is the largest social Q \& A community in China, this paper builds a relationship model of user heterogeneity and knowledge collaboration performance from the perspective of user interaction. The research conclusion has certain practical guidance value for the user management of the social Q \& A community.

\section{LITERATURE REVIEW AND FRAMEWORK 2.1 Social Q \& A Community}

The social Q \& A community serves to meet the user's knowledge demand. An open knowledge Q \& A community based on social network relationships for searching, sharing, and disseminating knowledge has both knowledge sharing and social functions [9], which is one of the important forms of knowledge communities in the network society. Its members gather together due to the certain topics, and achieve the sharing and collaboration of knowledge in the process of interaction [10]. Although the $\mathrm{Q} \& \mathrm{~A}$ community is a loose organization, it truly 
embodies the knowledge sharing and collaboration in the network society [6].

The social Q \& A community essentially addresses two key issues, namely how to find relevant knowledge and how to establish social connections with heterogeneous knowledge subjects [11]. At present, domestic and foreign scholars' research on the social Q \& A community focuses on three levels among which the user level comes first. Users are the main participants of the virtual community, and research on users has always been a hot spot. In the field of social Q \& A, scholars' focus on users mainly lies in the establishment of user role types and their relationships [12], user information behaviours (knowledge contribution, knowledge adoption, knowledge search, public editor and so on) [13], user social network, etc. In addition, some scholars have studied the users of the social Q \& A community from both the individual level and the group level; secondly, the content level. The present research mainly focuses on the quality of knowledge contributed by users in the community [8], the efficiency of knowledge ordering and the evolution of social systems [14], the evaluation of community information quality [15], knowledge aggregation and theme discovery [8]; and finally, the technical level. This level involves user question recommendation method based on big data technology [1], keyword extraction method [16], etc.

\subsection{User Interactivity}

Jiang et al. [17] believes that knowledge heterogeneity affects knowledge growth in two ways: first, it affects the rate of knowledge growth by affecting network knowledge heterogeneity; second, the correlation between knowledge stock and knowledge affects knowledge growth, that is user interaction. Interactivity is a major feature of Internet platform communication, and its essence is the degree of user involvement in knowledge interaction [4]. Hoffman et al. [18] divided the interactivity into two categories: machine interaction and interpersonal interaction. On this basis, $\mathrm{Wu}$ and Jin [19] define machine interaction in the context of online communities as interaction with the media, namely, direct interaction about information between users such as browsing, searching, sending and so on; while interpersonal interaction is defined as sharing values, exchanging information, and maintaining interpersonal relationships with others through the platform and computer. Nambisan et al. [20] divided user interaction in the virtual community into three dimensions: product content interaction, member cognitive interaction (identification) and interpersonal interaction. Wang et al. [21] also divided interaction into three dimensions: twoway communication, customer participation, and joint problem solving. Li et al. [22] divided the virtual brand community interaction into human-machine interaction, content interaction and social interaction.

This paper refers to the views of the above scholars and divides the user interaction in the social Q \& A community into machine interaction and interpersonal interaction. Among them, interpersonal interaction refers to the behaviours of users directly or indirectly interacting with other users in the social Q \& A community, such as asking or answering questions, publishing articles, and participating in public editing, etc.; machine interaction refers to the passive silent behaviours of users in the community, such as paying attention to topics, questions, columns, etc.

\subsection{User Heterogeneity and Knowledge Collaboration Performance}

Due to the unique value of heterogeneous resources in the growth and development of individuals and enterprises [23], the concept of user heterogeneity (UH) has become an important topic of common concern among academic and business communities. User heterogeneity can be defined from multiple dimensions, such as personality variables, demographic variables, cognitive style, knowledge, expertise, and functional roles. Some scholars believe that the most important differences in the heterogeneity dimensions are social heterogeneity and information or functional heterogeneity. Social genre heterogeneity refers to differences in team members in terms of gender, age, ethnicity and other demographic characteristics, and information or functional heterogeneity differences rely on the team members' educational background, functional background or professional experience [24]. In the context of a social Q \& A community, user heterogeneity refers to the differences of users in any individual traits, including educational background, industry background, knowledge field, and work place etc. These traits lead them to form perceptions of differences between individuals [6].

Knowledge collaboration performance is an indicator to measure the results of user interaction behaviours in knowledge communities. Different types of community users have different demands, thus the knowledge collaboration performance has different evaluation methods. Gao et al. [25] believes that in the field of communication-oriented social networks, the attention gained by users and the reposting of articles are the criteria for measuring the performance of knowledge collaboration. In the Q \& A community, Burghardt et al. [26] believe that the quality of answers is an important criterion for "good or bad". In this community, the substantial and accurate answers are more likely to be recognized by users. It can be seen from this that although knowledge performance in different contexts is different, the view of "relying on user evaluation of knowledge" has been widely recognized by scholars.

Regarding the relationship between heterogeneity and collaboration performance of knowledge, most scholars believe that the level of knowledge heterogeneity will affect the knowledge innovation performance of enterprises, and there is an inverted U-shaped nonlinear relationship between them [27] (As shown in Fig. 1). Similar conclusions have also been verified in the study of entrepreneurial teams. Qiu and Wang [14] discussed the effects of team heterogeneity and team interaction on the efficiency of knowledge ordering from the perspective of knowledge heterogeneity and experience heterogeneity. Song and Guo [28] also studied the relationship between heterogeneity and sales performance of online retailers from the perspective of online reviews. The research shows that the higher the heterogeneity of online reviews is, the more negative adjustment there will be that affects the 
relationship between online review features and online retailers. The lower the heterogeneity of online reviews is, the more positive the regulatory effect is.

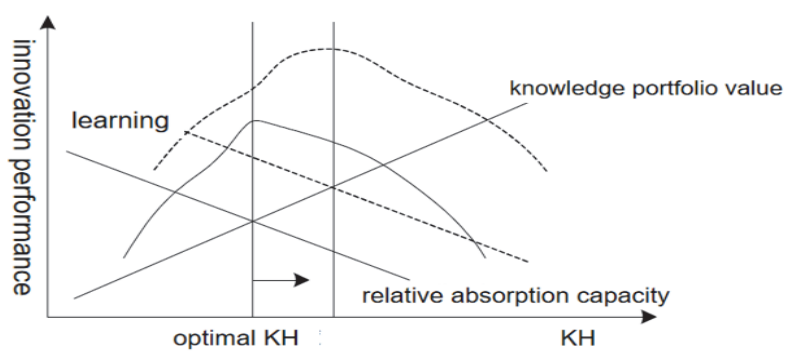

Figure 1 The relationship curve between knowledge heterogeneity $(\mathrm{KH})$ and knowledge innovation performance

The influence of knowledge (or user) heterogeneity on knowledge collaboration performance requires a certain path or mechanism, but the mechanism or path for the conversion of user heterogeneity into knowledge collaboration performance in the social Q \& A community is still unknown. In addition, the existing research has paid too much attention to the relationship between knowledge heterogeneity and performance at the enterprise level, and has ignored the research on the social Q \& A community situation; in the existing research, the inverted U-shaped nonlinearity relationship between the heterogeneity and knowledge collaboration performance has been verified at the level of the physical organization, and the situation of the virtual knowledge community has not been verified.

Based on these, this paper proposes a model for the conversion of user heterogeneity to knowledge collaboration performance in a socialized Q \& A community environment (see Fig. 2), exploring the relationship between user heterogeneity, interactivity, and knowledge collaboration performance, in order to provide certain references for the management of the community and the improvement of the collaboration performance.

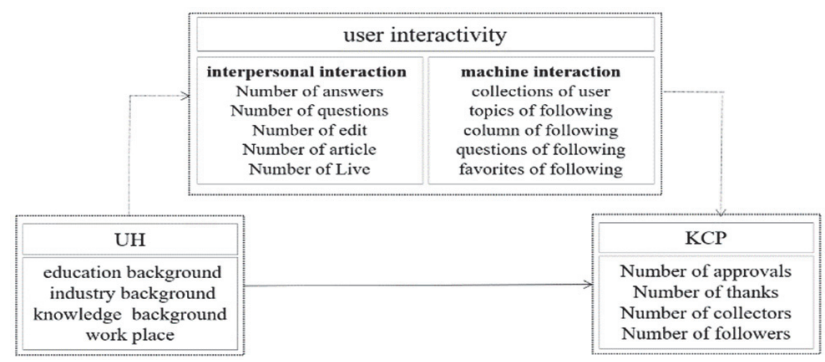

Figure 2 The research model.

\section{RESEARCH METHODOLOGY 3.1 Research Hypothesis}

User heterogeneity and knowledge collaboration performance. Rodan and Galunic [29] believe that knowledge heterogeneity involves the diversity of knowledge, skills and expertise available to team members. Heterogeneity has a certain impact on knowledge collaboration performance. As is mentioned earlier, the inverted U-shaped nonlinear relationship between them has been verified at the level of the entity organization. The heterogeneity of users in the social Q \& A community is mainly reflected in the heterogeneity of knowledge, and its relationship with performance has a similar pattern. Therefore, the following hypothesis is made:

H1: There is an inverted U-shaped relationship between user heterogeneity and knowledge collaboration performance in the $\mathrm{Q} \& \mathrm{~A}$ community. As user heterogeneity rises, knowledge collaboration performance first rises and then declines.

User heterogeneity and user interaction. Social interaction is a natural instinct of gregarious human beings. Purkhardt [30] believes that as long as social connections exist, there will be group cohesion. Even if the personnel are randomly assigned, partiality within the group will also occur. In the social Q \& A community, that means as long as there are links between user social connections (platforms and enough users), group interaction will occur even if the personnel are unfamiliar. Hoffman et al. [18], $\mathrm{Wu}$ and Jin [19] divide interactivity into interpersonal interaction and machine interaction. Interpersonal interaction is the interaction between users and other users in the medium of Q \& A community, which is the source of community knowledge innovation. machine interaction is the interaction between users and the platform, which reflects the user's attention to community members or community knowledge.

The social Q \& A community is an open knowledge community, and any users with knowledge demand or willingness to contribute can join it. And the support of internet technology enables it to achieve interaction and communication across time, space and other levels, which builds a bridge for group interaction. The purpose of the social Q \& A community, nevertheless, is not simply to gather the heterogeneous users, but also to promote the communication and interaction of heterogeneous users in the community [31]. Some studies have pointed out that the reason for knowledge community user interaction lies in seeking, contributing knowledge or establishing community connections from heterogeneous community users [32]. The higher the user heterogeneity in the community, the more users there are with different knowledge backgrounds, industry backgrounds, hobbies, and even work locations, correspondingly, the more "partners" a user has when participating in community interactions. This directly affects the activeness of users' participation in interactions. On the other hand, the increase in the heterogeneity of community users also increases the difficulty of users in choosing a certain knowledge requirement, which may affect the user's activity. Therefore, the following hypotheses are made.

H2a: There is an inverted U-shaped relationship between user heterogeneity and the interpersonal interactivity of community members. As user heterogeneity increases, interpersonal interactivity increases first and then decreases;

$\mathrm{H} 2 \mathrm{~b}$ : There is an inverted U-shaped relationship between user heterogeneity and community members' machine interaction. As user heterogeneity increases, machine interaction increases first and then decreases.

User interaction and knowledge collaboration performance. User interaction is the guarantee for the survival and development of the community, which can enhance the community's knowledge collaboration performance. As far as users are concerned, user interaction not only can meet the knowledge needs, it also 
can enhance the status and reputation of individuals in the virtual community (getting approval and gratitude), and at the same time establish community connections based on knowledge interests [33], such as getting their own followers. For the community, user interaction can continuously input new knowledge and opinions for the community to build a community social network, and expand the user scale via the platform's network. Therefore, the following hypotheses are made:

H3a: Interpersonal interaction positively affects knowledge interaction performance;

$\mathrm{H} 3 \mathrm{~b}$ : Machine interaction positively affects the knowledge interaction performance.

Mediating effect of user interaction. Heterogeneous knowledge resources are only the basis of knowledge sharing in the social Q \& A community which does not directly produce innovative reference, but relies on certain paths and intermediaries [14]. User interaction in the virtual community often promotes the collision of different types of knowledge, allowing that originally dispersed heterogeneous knowledge to be reorganized, merged and integrated. However, in the social Q \& A community, how does the user's heterogeneous knowledge "interact" into knowledge collaboration performance? At the enterprise level, organizational learning is the formation methodology of organizational knowledge, organizational memory, and organizational practices, and can also promote the knowledge and ability sharing among members. It is an important intermediary variable between heterogeneous knowledge resources and corporate performance [10]; although there is no explicit concept of organizational learning, there is objective learning and communication between users [34]. Therefore, this paper believes that, similar to organizational learning, user interaction plays a mediating role between user heterogeneity and knowledge collaboration performance. Hence the following hypotheses are made:

H4: Interpersonal interaction plays a mediating role between user heterogeneity and knowledge collaboration performance;

H5: Machine interaction plays a mediating role between user heterogeneity and knowledge collaboration performance.

\subsection{Research Data}

Sample selection and data processing. This article selects Zhihu as a typical research object. Zhihu was established in January 2011. And as of 2018, the number of users exceeded 220 million. It has become the most representative social Q \& A community in China. With the slogan "Share your knowledge, experience and insights with the world", Zhihu is a real online Q \& A community with a friendly and rational atmosphere, connecting elites from all walks of life. Users share each other's professional knowledge, experience and insights, and continuously provide high-quality information for the Chinese internet.

This paper is centered on the users of Zhihu by collecting relevant data which is open to the platform by stages. The data collection lasted for 53 weeks from September 2018 to October 2019. And the SQL Server 12 database and SPSS software were used to process the data. In the natural language processing process of Zhihu, the heterogeneity is calculated by measuring the similarity between the index texts. In the calculation process, we firstly vectorized the collected text to construct a word set model and a word generation model, and then calculated the Euclidean distance between the text vectors to quantify the degree of heterogeneity between the texts. For digital data, it is normalized in order to facilitate subsequent analysis.

Variable measurement. User heterogeneity (UH): the personal information provided by users of Zhihu on the platform includes knowledge information such as education background, industry background, knowledge fields, contributed knowledge and demographic information such as gender, age, and work place. Due to the user's protection of personal privacy, demographic information may be seriously missing, or its authenticity needs to be considered. After comprehensively considering the availability of data and research demand, the paper measures user heterogeneity from four dimensions: education background, industry background, knowledge background, and work place.

User interactivity: Dialogue and interaction are basic activities on social media. For a knowledge-based community, although the number of user interactions can represent the level of user interaction to a certain extent, the quality of the interaction itself, i.e., the quality of the question and answer, is also an important criterion for evaluating knowledge interaction [26]. This paper not only measures user interaction quantitatively, but also focuses on the quality of user interaction, that is, the accuracy of knowledge provided by users and their relevance to topics. As is mentioned above, in the social Q \& A community, the user interactive behavior can be divided into interpersonal interaction and machine interaction based on whether there is direct knowledge exchange. According to the user interactive information on the platform, the article believes that interpersonal interaction can be valued by the number of user answers, questions, public editors, articles, and live numbers, and machine interaction is measured by the user's collections, topics, columns, questions, and favorites.

Knowledge collaboration performance (KCP): Zhihu is a social Q \& A platform based on the operation of user, relationship and content. The questions are mostly subjective to a certain question, so there is no standard answer. And the answer is based on user experience and knowledge, to meet the knowledge needs of all users in the entire community, so the evaluation of other users in the community can roughly reflect the quality of the answer. Therefore, this paper believes that, for a social Q \& A community, knowledge collaboration performance can be reflected by user evaluation of knowledge. Specific evaluation indicators include the number of approvals, the number of thanks, the number of favorites, and the number of followers.

\section{RESEARCH RESULTS}

This paper uses SPSS2.0 software to test the research hypothesis through hierarchical regression. The results are shown in Tab. 1. As we can see from model 1, the coefficient between user heterogeneity and knowledge collaboration performance is not significant, indicating that 
there is no linear relationship between them. To further explore the relationship between them, the square term of user heterogeneity is introduced, as shown in model 2. Compared with model 1 , model 2 has a better fitting effect (adjusting $R^{2}$ to increase from 0.019 to 0.135 ) and has stronger explanatory power. For the test of inverted Ushaped model, most of the existing studies are based on the coefficient of the square term of the independent variable, and there is a certain deviation. According to the view of Richard et al. [35], the test result is that the inverted $U$ shape needs to meet three conditions. First, the coefficient of the square term of the explanatory variable is negative. Second, the slope of the curve takes a positive value on the left side but a negative value on the right. Third, the inflection point of the curve is within the range of the explanatory variables. According to the regression results, the coefficient of the square term of user heterogeneity is negative ( -7.649$)$, and the coefficient of user heterogeneity is 12.315 , both of which are significant within the $95 \%$ confidence interval, and can be seen from the model fitting diagram (Fig. 3). The inflection point of the curve is within the range of user heterogeneity, indicating that there is a significant inverted U-shaped relationship between user heterogeneity and knowledge collaboration performance. Hypothesis H1 is verified.

Model 3 verifies the influence of interpersonal interaction and machine interaction on knowledge collaboration performance. According to the results, the coefficients of interpersonal interaction and machine interaction are both positive and significant at different levels of significance, indicating that both of interpersonal interaction and machine interaction positively affect knowledge collaboration performance. Hypotheses $\mathrm{H} 3 \mathrm{a}$ and $\mathrm{H} 3 \mathrm{~b}$ are verified.

Table 1 The regression result

\begin{tabular}{|c|c|c|c|c|c|}
\hline \multirow[t]{2}{*}{ Variable } & \multicolumn{3}{|c|}{$\begin{array}{l}\text { Knowledge collaboration } \\
\text { performance }\end{array}$} & \multirow{2}{*}{$\begin{array}{c}\text { Interpersonal interaction } \\
\text { Model } 4\end{array}$} & \multirow{2}{*}{$\begin{array}{c}\text { Machine interaction } \\
\text { Model } 5 \\
\end{array}$} \\
\hline & Model 1 & Model 2 & Model 3 & & \\
\hline User heterogeneity & -0.130 & $12.315 * *$ & & $9.160 * *$ & $2.432 * *$ \\
\hline Square of user heterogeneity & & $-7.649 * *$ & & $-5.689 * *$ & $-1.510 * *$ \\
\hline Interpersonal interaction & & & $0.558 * *$ & & \\
\hline Machine interaction & & & $3.021 * * *$ & & \\
\hline$R^{2}$ & 0.001 & 0.168 & 0.284 & 0.173 & 0.170 \\
\hline Adjusted $R^{2}$ & 0.019 & 0.135 & 0.262 & 0.140 & 0.137 \\
\hline$F$ & 0.026 & 5.041 & 16.290 & 5.239 & 5.138 \\
\hline
\end{tabular}

Note: *** means $p<0.01, * *$ means $p<0.05, *$ means $p<0.1$

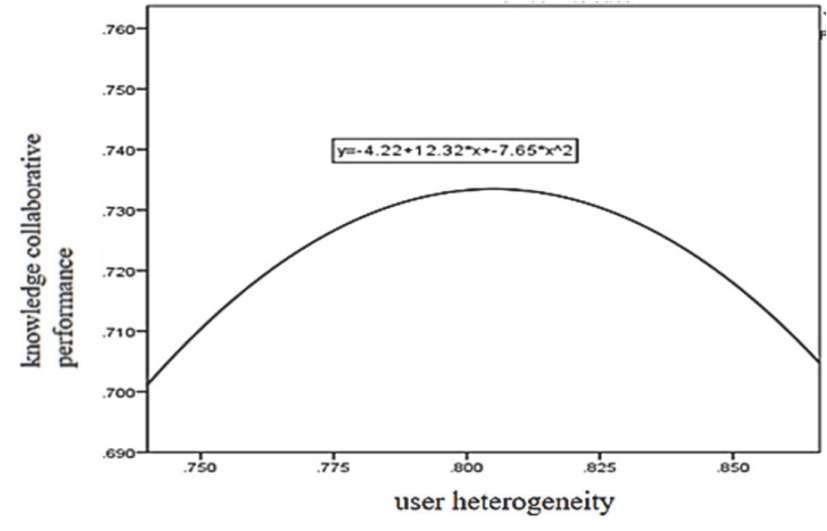

Figure 3 Fitting diagram of relationship between user heterogeneity and knowledge collaboration performance.

Model 4 verifies the impact of user heterogeneity on interpersonal interactivity. The results show that the coefficient of the square term of user heterogeneity is negative ( -5.689$)$ and the coefficient of user heterogeneity is 9.16 , both of which are within the $95 \%$ confidence interval, indicating that there is a significant inverted Ushaped relationship between user heterogeneity and interpersonal interactivity, assuming that hypothesis $\mathrm{H} 2 \mathrm{a}$ is proven. Model 5 verifies the impact of user heterogeneity on machine interaction. The results show that the coefficient of the square term of user heterogeneity is negative $(-1.51)$, the coefficient of user heterogeneity is 2.432 , both are significant within the $95 \%$ confidence interval, indicating that there is also a significant inverted $\mathrm{U}$ between user heterogeneity and machine interaction, assuming that hypothesis $\mathrm{H} 2 \mathrm{~b}$ is verified.

This paper uses the hierarchical regression method to further verify the mediating effect of the user's interpersonal interaction and machine interaction. According to the results of Model 4 and Model 3, there is a significant inverted U-shaped relationship between user heterogeneity and interpersonal interactivity, and there is a significant positive relationship between interpersonal interactivity and knowledge collaboration performance. The results after adding intermediary variables to interpersonal interaction are shown in Model 6. User heterogeneity and its square coefficient are significant at $\mathrm{p}$ $<0.05$, and the coefficient of interpersonal interaction is significant within the $99 \%$ confidence interval, indicating that the mediating effect of interpersonal interaction is significant, and hypothesis H4 is verified. Similarly, Model 7 introduces machine interaction as an intermediary variable. From the analysis results, it can be seen that there is a significant inverted U-shaped relationship between user heterogeneity and machine interaction. The coefficient of machine interaction in Model 7 is significant at the $99 \%$ confidence level, and the square term of user heterogeneity is not significant, which indicates that the mediating effect of machine interaction between user heterogeneity and knowledge collaboration performance is not significant. Hypothesis H5 has not been verified.

\begin{tabular}{|c|c|c|}
\hline \multirow[t]{2}{*}{ Variable } & \multicolumn{2}{|c|}{$\begin{array}{l}\text { Knowledge collaboration } \\
\text { performance }\end{array}$} \\
\hline & Model 6 & Model 7 \\
\hline User heterogeneity & $0.242 * *$ & -0.117 \\
\hline Square of user heterogeneity & $-0.149 * *$ & 0.072 \\
\hline Interpersonal interaction & $1.371 * * *$ & \\
\hline Machine interaction & & $5.113 * * *$ \\
\hline$R^{2}$ & 0.324 & 0.331 \\
\hline Adjusted $R^{2}$ & 0.317 & 0.323 \\
\hline$F$ & 50.972 & 42.018 \\
\hline
\end{tabular}




\section{DISCUSSION AND CONCLUSION}

\subsection{Discussion}

This paper explores the relationship between user heterogeneity and knowledge collaboration performance, as well as the mediating effect of user interaction, based on the user's knowledge behaviours of Zhihu, the largest social Q \& A community in China. The main topics we can discuss are as follows.

First, in the social Q \& A community, there is a significant inverted U-shaped relationship between user heterogeneity and knowledge collaboration performance. With the increase of user heterogeneity in terms of educational background, work background, knowledge domain, and work place, the knowledge collaboration performance increases first and then decreases. This conclusion is similar to the knowledge heterogeneity and knowledge collaboration performance discussed by existing scholars at the entity organization level. It can be seen that in the social Q \& A community, before the user heterogeneity reaches the threshold, increasing the heterogeneity is conducive to improve the performance. However, when the knowledge heterogeneity is too large, it often leads to a series of misunderstandings, reducing the speed of decision-making, affecting emotions, and even affecting the user's accurate grasp of knowledge as a result of knowledge overload [36], for the community, it will reduce the overall knowledge collaboration performance.

Second, there is a significant inverted U-shaped relationship between user heterogeneity and interpersonal interactivity and machine interactivity. In addition, interpersonal interaction has a significant mediating effect between user heterogeneity and knowledge collaboration performance, while the mediating effect of machine interaction is not significant. In the social $Q$ \& $A$ community, the reason of producing knowledge collaboration performance among heterogeneous users not only relies on the interaction opportunities for knowledge demanders and knowledge providers (users, platforms) to provide cross-time and cross-regional interaction, but also relates to the platform for users instant interaction provided by the community. Research shows that user-to-user interaction has a significant impact on group creativity [37]. Increased connections and interactions among users in the virtual community can encourage community users to generate higher willingness to participate in innovation, thereby promoting individual innovation participation of users [38].

Therefore, different users can affect knowledge collaboration performance through interpersonal interaction, that is, interpersonal interaction has a significant mediating effect between user heterogeneity and knowledge collaboration performance. The machine interaction is only the interaction between the user and the platform, and the knowledge flow is one-way. The user obtains his own useful information or knowledge through browsing, collecting, following, and other behaviours, and does not produce direct knowledge collaboration results which is one of the reasons why the mediating effect between heterogeneity and knowledge collaboration performance is not significant. In fact, the impact of machine interaction on the performance of knowledge collaboration is indirect, that is, it provides hidden value to the community through network externalities, attracts more users to participate, and thus indirectly contributes to knowledge collaboration [39].

The theoretical contribution of this paper is that, first of all, it reveals the inverted U-shaped relationship between user heterogeneity and knowledge collaboration performance in online knowledge communities, indicating that there is an optimal point for the user heterogeneity. Secondly, in the previous literature on user interaction, it is rare to distinguish between interpersonal interactivity and machine interaction. This paper distinguishes them and the research conclusions show that interpersonal interactivity has a more significant mediating effect.

\subsection{Research Conclusion and Implications}

In the social Q \& A community, user heterogeneity expands the breadth of knowledge interaction between users, and user interaction deepens the depth of knowledge interaction between users on the basis of heterogeneity [40], both of them have a significant impact on the improvement of collaboration performance. However, at this stage, there is certain heterogeneity of users for the social Q \& A community represented by Zhihu, the heterogeneity of existing users is not well converted synergistic performance for community knowledge due to the excessive number of "silent" users. Based on the above research, this paper proposes the following management implications.

Firstly, according to the research results, there is an inverted U-shaped relationship between user heterogeneity and knowledge collaboration performance. Increasing the heterogeneity of users is conducive to improving knowledge collaboration performance in the early stages of community development. When the threshold is reached, the platform can subdivide users into different groups by technical means, which cannot only ensure the knowledge flow of the platform, but also improve the overall performance by improving the knowledge collaboration performance of each group. Therefore, the community should strive to attract users' participation in various ways, and at the same time, strengthen technology research and development, make full use of big data technology to ensure that the platform can guide users to join topics or groups that match their own knowledge or interests in a timely manner through topic push according to the user's background, knowledge search, interaction and other behaviours, postpone the arrival of the community's overall heterogeneity threshold to improve knowledge collaboration performance.

Secondly, interpersonal interactivity has a significant mediating effect between user heterogeneity and knowledge collaboration performance, that is, the social Q \& A community can improve the user heterogeneity into knowledge collaboration performance by enhancing interaction among users. Therefore, the social Q \& A community should take various measures to stimulate the enthusiasm of existing users to participate in interpersonal interactions. For users with a relatively high level of activity, the platform should maintain or enhance its interactivity through community rewards, level upgrades, and the establishment of strict regulatory measures to ensure its high-quality interaction. For new users or those 
with low activity, the platform can use big data technology to build discussion groups on different topics to attract users' participation based on the real-time dynamics of the outside world and the users' real-time interests or topics. In addition, inviting well-known figures in different fields to regularly conduct online activities can also increase user participation.

\subsection{The Limitation and Future Direction}

The limitations of this paper are mainly including the following: first, the research data in this paper comes from the Zhihu database. Due to the platform's protection of user information, the data that can be obtained to reflect the heterogeneity of users is limited. Therefore, the measurement of user heterogeneity is not accurate enough; secondly, the time for data collection is not long enough, and some laws based on time trends may go undiscovered. Therefore, on the basis of existing data, the research team will continue to reasonably obtain platform user information, expand the user group, and do more detailed research.

\section{Acknowledgements}

Supported by the National Natural Science Fund Project (71572015); Supported by the Talent Selection Program of Beijing Union University (BPHR2017CS06); Supported by the Funding project of Beijing Philosophy and Social Science Research Program (17BGL033).

\section{REFERENCES}

[1] Duica, M., Florea, N., Duica, A., \& Gilmeanu, R. (2019). Improving The Relationships Between Organizations And Their Customers Using Digital Multichannel Communication And Mathematical Simulation. Economic Computation And Economic Cybernetics Studies And Research, 53(1), 265-280. https://doi.org/10.24818/18423264/53.1.19.17

[2] Galunic, D. C. \& Rodan, S. (1998). Resource recombination in the firm: Knowledge structures and the potential for Schumpeterian innovation. Strategic Management Journal, 19(1), 1193-1201 https://doi.org/10.1002/(SICl)10970266(1998120)19:12<1193::AID-SMJ5>3.0.C0;2-F

[3] Wei, J., Ying, Y., \& Liu, Y. (2014). The Decentralization of the R\&D Network, the Sequence of the Organizational Learning and the Innovation Performance: A Multi-case Study. Management World, 2, 137-152.

[4] Deng, S. L., Chen, X. Y., \& Fu, S. X. (2017). Exploring the Effect of Users' Information Need on Information Seeking in Social Q\&A: the Mediating Role of Affinity with SQA Communities. Information Science, 35(07), 3-8+15.

[5] Cooke, N. J., Gorman, J. C., Myers, C. W, et al. (2013). Interactive Team Cognition. Cognitive Science, 7(2), 255285. https://doi.org/10.1111/cogs.12009

[6] Fang, C. C. \& Zhang, J. T. (2018). Research of Users in Social Q\&A Communities: Commentary and Future Directions. Journal of Intelligence, 37(09), 185-193.

[7] Shah, C., Kitzie, V., \& Choi, E. (2014). Modalities, motivations, and materials-investigating traditional and social online Q\&A services. http://doi.org/10.1177/0165551514534140

[8] Tao, X., Zhang, X. X., \& Guo, S. L. (2019). Research of User-generated-answer Knowledge Aggregation and Topic
Discovery Service in Social Q\&A Community Based on DPCA. Information Studies: Theory \& Application, 42(06), 94-98+87.

[9] Duica, M., Florea, N., Duica, A., \& Gilmeanu, R. (2019), Improving The Relationships Between Organizations And Their Customers Using Digital Multichannel Communication And Mathematical Simulation. Economic Computation And Economic Cybernetics Studies And Research, 53(1), 265-280. https://doi.org/10.24818/18423264/53.1.19.17

[10] Zhou, Y. Y., Gao, S. L., \& Chen, J. B. (2019). A Study on Motivations of R\&D Team Participating in Knowledge Collaboration from the Perspective of Organizational Learning. Science and Technology Management Research, 39(02), 140-148.

[11] Yang, S. J. \& Chen, I. Y. (2008). A social network-based system for supporting interactive collaboration in knowledge sharing over peer-to-peer network. International Journal of Human.Computer Studies, 66(1), 36-50. http://doi.org/10.1016/j.ijhcs.2007.08.005

[12] Shi, Y. P., Yuan, X. L., \& Song, G. (2018). Analysis on Knowledge-sharing Behaviours Characteristics of Opinion Leaders in Social Q \& A Platform. Documentation. Information \& Knowledge, (06), 103-112. https://doi.org/10.1088/1475-7516/2018/06/012

[13] Fu, S. X., Chen, X. Y., \& Deng, S. L. (2017). Information Behavioural Transferring in a Social Q \& A Community: A Conceptual Model for Understanding Mechanism from Information Adoption to Sustained Information Seeking. Documentation. Information \& Knowledge, (04), 80-88.

[14] Qiu, J. N. \& Wang, J. X. (2018). The Impact of Group Heterogeneity on Knowledge Ordering Efficiency in Online Knowledge Communities. Journal of the China Society for Scientific and Technical Information, 37(04), 372-383.

[15] Jin, J., Yan, X. B., \& Li, Y. J., et al. (2016). How users adopt healthcare information: An empirical study of an online Q\&A community. International Journal of Medical Informatics, 86, 91-103. https://doi.org/10.1016/j.jimedinf.2015.11.002

[16] Voican, O. (2020). Using Data Mining Methods To Solve Classification Problems In Financial-Banking Institutions. Economic Computation And Economic Cybernetics Studies And Research, 54(1), 159-176. https://doi.org/10.24818/18423264/54.1.20.11

[17] Jiang, J. F., Zhang, L. W., \& Wang, Y. P. (2017). Driven Mechanism of Heterogeneity on Knowledge Growth withinlnno-Net. Systems Engineering, 35(11), 74-79.

[18] Hoffman, J. J., Hoelscher, M. L., \& Sherif, K. (2005). Social capital, knowledge management, and sustained superior performance. Journal of Knowledge Management, 9(3), 93100. https://doi.org/10.1108/13673270510602791

[19]Wu, Y. P. \& Jin, H. (2018). A Study on the Impact of Users' Interaction and Self-disclosure on Intention Behaviours. Journal of Technical Economics \& Management, (09), 2630.

[20]Nambisan, S. \& Baron, R. A. (2009). Virtual Customer Environments:Testing a Model of Voluntary Participation in Value Cocreation Activities. Journal of Product Innovation Management, 26(4), 388-406. https://doi.org/10.1111/j.1540-5885.2009.00667.x

[21] Wang, L. (2016). Research on the Quality of Knowledge Contribution and Knowledge Interaction in Social Q\&A Communities. Harbin Institute of Technology.

[22] Li, X. X., Guo, C., \& Yu, T. (2019). Effects of the Interactions of Brand Virtual Community on Consumer Brand Evangelism. Journal of Liaoning University (Philosophy and Social Sciences Edition), 47(04), 47-54.

[23] Peng, L. \& Kloeden, P. (2019). Impulsivity And Heterogeneity. Economic Computation And Economic 
Cybernetics Studies And Research, 53(4), 313-324. https://doi.org/10.24818/18423264/53.4.19.19

[24] Ren, Y., Chen, J., \& Riedl, J. (2016). The impact and evolution of group diversity in online open collaboration. Management Science, 62(6), 1668-1686. https://doi.org/10.1287/mnsc.2015.2178

[25] Gao, S., Ma, J., \& Chen, Z. (2014). Effective and Effortless Features for Popularity Prediction in Microblogging Network. Proceedings of the $23^{\text {rd }}$ International Conference on World Wide Web, ACM, 269-270. https://doi.org/10.1145/2567948.2577312

[26] Burghardt, K., Alsina, E. F., Girvan, M., et al. (2017). The Myopia of Crowds: Cognitive Load and Collective Evaluation of Answers on Stack Exchange. PloS one, 12 (3), e0173610. https://doi.org/10.1371/journal.pone.0173610

[27] Van de Vrande, V. (2013). Balancing your technologysourcing portfolio: How sourcing mode diversity enhances innovative performance. Strategic Management Journal, 34(5), 610-621. https://doi.org/10.1002/smi.2031

[28] Song, P. \& Guo, Q. Q. (2019). Study on Influencing Factors of Online Retailer's Operating Performance-Based on the Perspective of Online Comment Heterogeneity. Friends of Accounting, (12), 35-40.

[29] Rodan, S. \& Galunic, C. (2004). More than network structure:How knowledge heterogeneity influences managerial performance and innovativeness. Strategic Management Journal, 25(6), 541-562. https://doi.org/10.1002/smi.398

[30] Caroline, P. S. (2017). Transforming Social Representations: A Social Psychology of Common Sense and Science. Routledge. https://doi.org/10.4324/9781315725864

[31]He, L., He, D. Q., \& Zhang, C. Z. (2018). Survey on Social Question and Answer. Data Analysis and Knowledge Discovery, 2(07), 1-12. https://doi.org/10.1088/1475-7516/2018/07/001

[32] Zhang, Y. (2016). Research on Co-evolution of Knowledge and Social System Based on Individual Heterogeneity in OKC. Dalian University of Technology.

[33] Li, W. Y., Zhai, X. X., \& Xu, F. (2018). Research of the Impact of Interpersonal Relationship Motivation on Knowledge Sharing Behaviours in Visual Brand Communities: A Moderated Mediation Model. Management Review, 30(07), 89-99. https://doi.org/10.1088/1475-7516/2018/07/009

[34] Zhou, Y. Y., Gao, S. L., \& Chen, J. B. (2018). Motivations of $\mathrm{R} \& \mathrm{D}$ team participating in knowledge collaboration: A game analysis and empirical study. Journal of Interdisciplinary Mathematics, 21(4), 975-987. https://doi.org/10.1080/09720502.2018.1486278

[35] Richard, F., J., Haan, C. P., et al. (2015). Thinking about u: theorizing and testing $\mathrm{u}$ - and inverted $\mathrm{u}$-shaped relationships in strategy research. Strategic Management Journal. https://doi.org/10.1002/smj.2399

[36] Ni, X. D. (2010). Research on the Effect Mechanism of Knowledge Heterogeneity on Team Innovation. Enterprise Economy, (08), 57-63.

[37] Zhang, J. S., Li, H. G., \& Han, L. C. (2017). An Empirical Study of Influence of Virtual Community Interactivity on Iterative Innovation. Industrial Engineering and Management, 22(05), 128-134.

[38] Chang, Y. P., \& Zhu, D. H. (2011). Understanding social networking sites adoption in China: A comparison of preadoption and post-adoption. Computers in Human Behaviours, 27(5), 1840-1848. https://doi.org/10.1016/j.chb.2011.04.006

[39] Qiu, J. N., Zhang, Y., \& Xu, K. (2018). Research on the Coevolution of Knowledge and Social System Based on Individual Heterogeneity in OKC. Operations Research and Management Science, 27(05), 119-129.
[40] Liu, J. Y., Wang, Y., \& Lin, L. (2020). The Impact of User Innovation Behaviours on Enterprise Innovation Performance under OIC-the Perspective of Social Network. Science \& Technology Progress and Policy, 37(06), 128136.

\section{Contact information:}

Yingying ZHOU, $\mathrm{PhD}$ candidate

School of Economics and Management,

Beijing Forestry University,

Beijing 100083, China

E-mail: zyy6259@163.com

Jianbin CHEN, PhD

(Corresponding author)

School of Business, Beijing Union University,

Beijing 100025, China

E-mail: jianbin.chen@buu.edu.cn

\section{Shuli GAO, PhD}

School of Business, Beijing Union University,

Beijing 100025, China

E-mail: shuli.gao@buu.edu.cn

Mo CHEN, PhD

School of Business, Beijing Union University, Beijing 100025, China

E-mail: mo.chen@buu.edu.cn 